Proc. XII Int. School on Theoretical Physics — Symmetry and Structural Properties of Condensed Matter

\title{
Improvement of Solar Cells Efficiency through Detailed Characterization of $\mathrm{TCO} / \mathrm{TiO}_{2}$ Interface
}

\author{
P. KWAŚNICKI ${ }^{a, *}$ AND M. INGLOT ${ }^{b}$ \\ ${ }^{a}$ Research and Development Centre for Photovoltaics, ML System S.A., Zaczernie 190 G, 36-062 Zaczernie, Poland \\ ${ }^{b}$ Department of Physics and Medical Engineering, Rzeszów University of Technology, \\ al. Powstańców Warszawy 6, 35-959 Rzeszów, Poland
}

\begin{abstract}
In this work we present a detailed characterization of the interface $\mathrm{TCO} / \mathrm{TiO}_{2}$ layer in order to improve the final efficiency of the solar cell. High resolution SEM measurements done in cross-section configuration as well as optical measurements were performed to obtain a topographic and optical image of measured layer.
\end{abstract}

DOI: 10.12693/APhysPolA.132.179

PACS/topics: photovoltaic, solar cell, dye sensitized solar cell, quantum dots solar cell, $\mathrm{TCO} / \mathrm{TiO}_{2}$ interface, $\mathrm{SEM}$

\section{Introduction}

Solar power can easily meet the demands of electrical power for whole world if only we could decrease the power to price ratio for solar panels [1]. One of the possible way is to reduce the production cost and as so the third generation of solar cells such as dye sensitized solar cell (DSSC) [2] and quantum dots solar cell (QDSC) [3] were introduced. Nonetheless there are still some issues to be overcome. The typical structure of DSSC and its simple adaptation for QDSC is based on glass with transparent conductive oxide (TCO) layer and then using screen printing as a cheap and fast method a mesoporous layer of $\mathrm{TiO}_{2}$ is deposited. This process although being used very often especially for DSSC does not provide a good interface quality of heterostructure of TCO layer and photoanode layer. So far the particles size of $\mathrm{TiO}_{2}$ was mainly studied in order to increase the adsorption of dye [4], or diffusion and recombination in dye-sensitized $\mathrm{TiO}_{2}$ solar cells [5]. In our work we mainly focus on increase of the charge transfer in order to increase final efficiency.

\section{Experimental details}

A series of samples were prepared in order to measure the interface of $\mathrm{TCO}$ and titanium dioxide $-\mathrm{TiO}_{2}$ layers. All samples were prepared on glass substrate of a thickness in range of $2.2 \mathrm{~mm}$. The TCO layer was made using chemical vapor deposition (CVD) and pyrolytic coating process. A smooth TCO glass with a low haze value is required for the PV application. Next on the top of TCO layer a transparent mesoporous layer of $\mathrm{TiO}_{2}$ was deposited using screen printing method. For this we use a paste of $\mathrm{TiO}_{2}$ particles mixed with organic material to ensure good rheological parameters needed during the printing process. After each printing process

*corresponding author; e-mail: pawel.kwasnicki@mlsystem.pl the samples were dried and annealed in a well-established temperature profile process. The thickness of the layers was measured using ellipsometry spectroscopy or optical profilometer and it was found for TCO to be in range of $600 \mathrm{~nm}$ and for $\mathrm{TiO}_{2}$ around $2.5 \mu \mathrm{m}$. To improve the charge transfer confection we propose to decrease the $\mathrm{TiO}_{2}$ particles size in order to obtain better interface between $\mathrm{TCO} / \mathrm{TiO}_{2}$ layers.

Characterization of the morphology was done using scanning electron microscopy (SEM). Measurements were done in both top-view and cross-section configuration. In order to obtain the best resolution we used in various detectors as well as deceleration beam feature. Secondary electron detector (SED) was used to obtain a topographic SEM image. SED images have high resolution that are independent of the material and are acquired from inelastically scattered electrons close to the surface, nonetheless no material composition information is available. For the additional information a backscatter electron detector (BSD) which detects elastically scattered electrons was used. For the element composition determination an energy dispersive X-ray spectroscopy (EDS) was used.

$\mathrm{X}$-ray diffraction (XRD) was used in order to measure the size of the $\mathrm{TiO}_{2}$ nanoparticles before and after the milling process as well as for the characterization of the crystalline structure. To calculate the diameter of the particles we applied the Sherrer equation.

Solar cell spectral response (SCSR) to measure the internal and external quantum efficiency in a function of wavelength. Measurements were done using single monochromator and a xenon/quartz halogen light source, launched onto the sample under test, giving coverage over the spectral range 350-2500 $\mathrm{nm}$ with spectral resolution of $0.1 \mathrm{~nm}$. All measurements were done in room temperature.

\section{Results and discussion}

The first step was to measure the external quantum efficiency (EQE) for the samples prepared using standard $\mathrm{TiO}_{2}$ paste with bigger particles size. The initial size of 
the $\mathrm{TiO}_{2}$ particles was in range of $0.29 \mu \mathrm{m}$, which is a typical commercially available size for $\mathrm{TiO}_{2}$ particles.

Figure 1 shows the EQE versus wavelength for four different samples.In this case the value measured for all four samples was varied from 1.5 up to $2.3 \%$. In order to obtain clear image of the interface of $\mathrm{TCO} / \mathrm{TiO}_{2}$ we decided to reduce the amount of absorber. The low EQE was due to low amount of absorber that was used. The differences between samples are related to the type of absorber. All samples then were cleaved in order to perform SEM measurements in cross-section configuration. High resolution SEM measurements showed a gap between TCO and $\mathrm{TiO}_{2}$ layer. Although in some places the $\mathrm{TiO}_{2}$ crystals are linked to the TCO layer mostly the gap is notable. We estimated the size of the gap to vary from few $\mathrm{nm}$ to even tens of $\mathrm{nm}$ which could hardly decrease the charge transfer between these two layers.

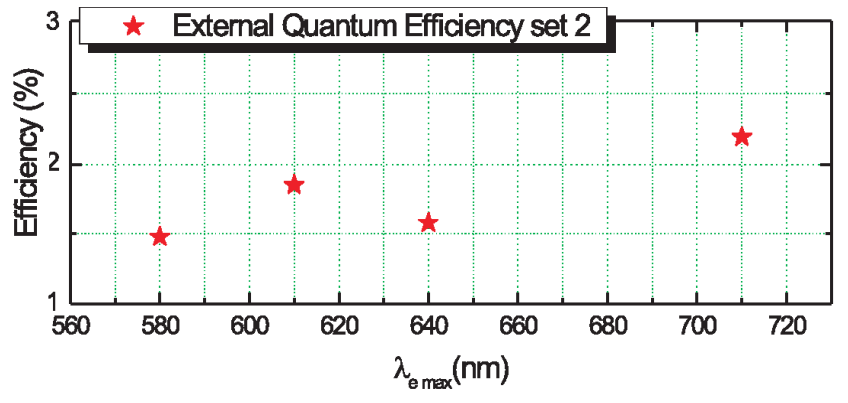

Fig. 1. External quantum efficiency vs. wavelength for four different samples.

Figure 2 presents the SEM image collected using high resolution scanning electron microscopy. Taking into account that $\mathrm{TiO}_{2}$ layer is a porous film, in order to overcome the surface charge accumulation effect we had to tune the accelerating voltage and set on low value and use the deceleration beam option. Looking from the top we can see the absorber layer (not homogeneous in thickness film), then a $\mathrm{TiO}_{2}$ layer, lower TCO and finally the substrate which in this case was glass sheet. One interesting point was noticed in the interface glass/TCO layer. One can clearly see a line in the bottom part of the TCO layer. This is most probably due to the CVD process used for deposition of the TCO film. Nonetheless, because of the fact that this appears in lower region of TCO layer, no negative impact on the efficiency was noticed. One of the issue we meet was related to the $\mathrm{TCO} / \mathrm{TiO}_{2}$ interface. Low adhesion of titania layer and a gap between these two films is one of the main reason why we observe low efficiency for final solar cell.

In addition the photo catalytic efficiency of titania $\left(\mathrm{TiO}_{2}\right)$ depends highly on particle size and surface area of the material [6], the efficiency of $\mathrm{TiO}_{2}$ for DSSC is highly depending on particle size and surface area [7]. So far nanosized $\mathrm{TiO}_{2}$ was fabricated using sol-gel, sputtering, combustion flame, and thermal plasma. Although the sol-gel method is considered as a suitable method to synthesize ultrafine particles, this method needs a large
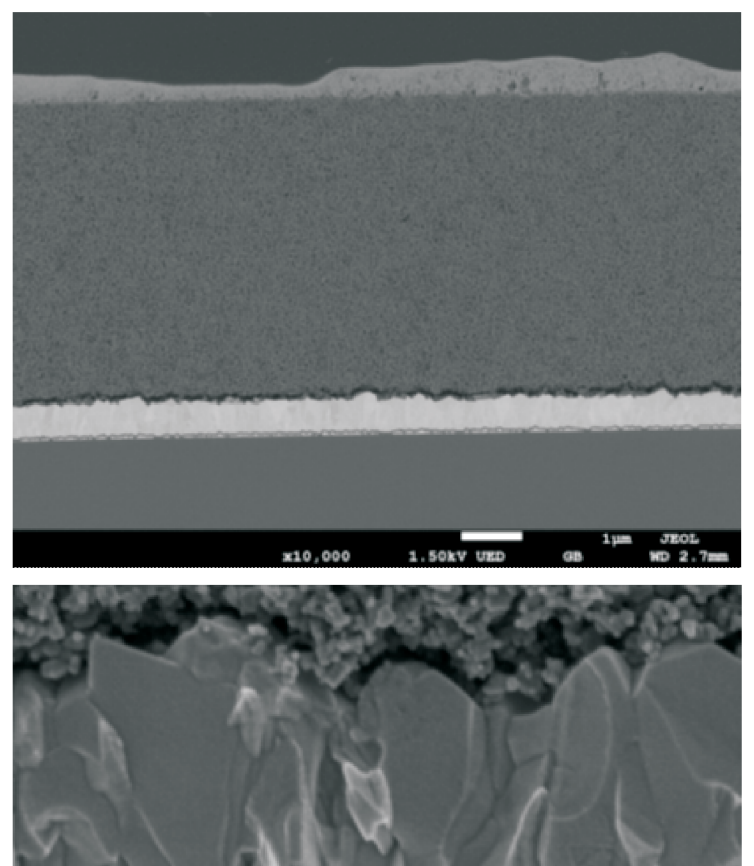

Fig. 2. High resolution image of a stack layers (view from the bottom: glass $/ \mathrm{TCO} / / \mathrm{TiO}_{2} /$ absorber layer/air) and below zoom on a $\mathrm{TCO} / \mathrm{TiO}_{2}$ interface region.

quantity of solution, longer processing time and heat treatment for crystallization since amorphous $\mathrm{TiO}_{2}$ has a very little photocatalytic activity and so this considerably increases the cost of the solar cell. In order to overcome this problem and to not increase the cost of the DSS cell we propose to reduce the size of the $\mathrm{TiO}_{2}$ particles by using high energy grinding process. By this process we could easily obtain the $\mathrm{TiO}_{2}$ particles size of diameter below $20 \mathrm{~nm}$ while still keeping suitable parameter of nanoparticles. The only parameter that determines the final result i.e. the size it grinding time, so it is easy to control the size by adjusting process duration. Changing the size of the particles of the $\mathrm{TiO}_{2}$ layer we could successfully improve the interface between TCO and $\mathrm{TiO}_{2}$ layers.

Image 3 shows the interface between TCO and $\mathrm{TiO}_{2}$. This time the $\mathrm{TiO}_{2}$ layer was made using smaller particles. One can notice reduction of the gap between these two layers. This time the gap between two layers do not exceeds few $\mathrm{nm}$ and in most of the region it is hardly noticeable. We do not only reduce the gap between TCO and $\mathrm{TiO}_{2}$ layers but also significantly increase the contact region. We also notice improvement in terms of the adhesion, now $\mathrm{TiO}_{2}$ film is much stronger connected to the TCO layer. The main point though, was to improve the charge transfer between this heterostructure.

This was achieved which is presented in Fig. 4. External quantum efficiency is plotted against wavelength. Red stars correspond to the results obtained for samples with bigger $\mathrm{TiO}_{2}$ particles size and blue stars are for the layer with $\mathrm{TiO}_{2}$ after grinding process. One can easily 

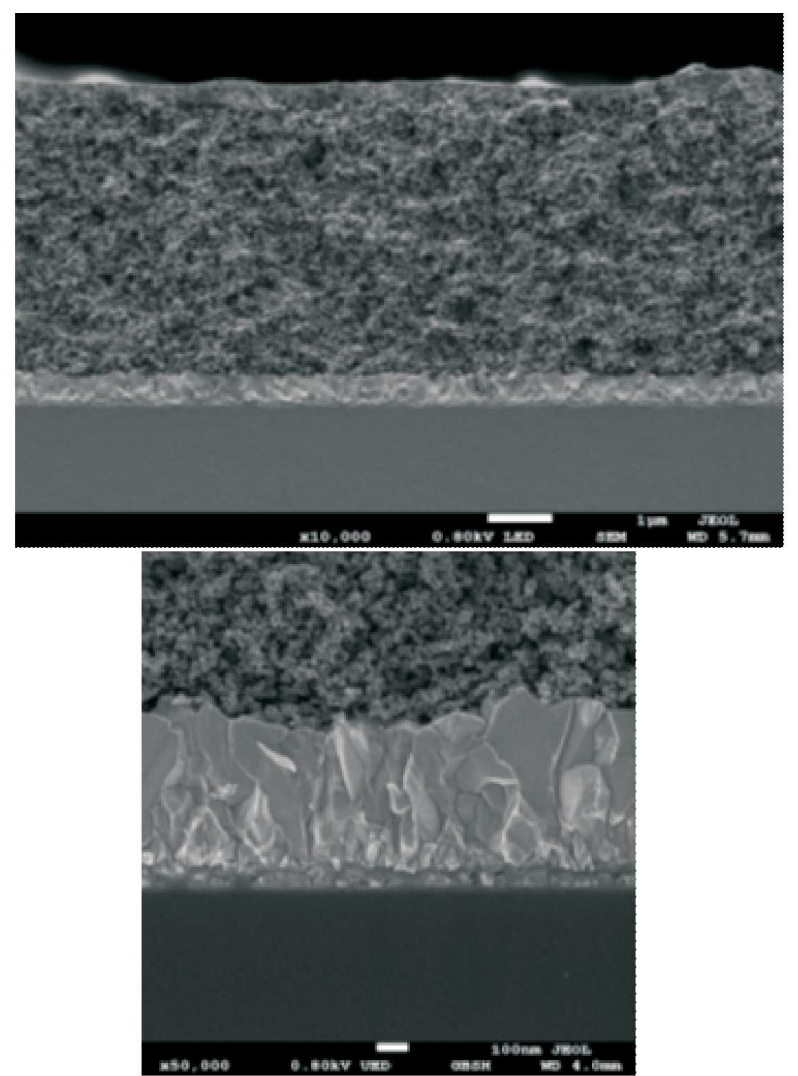

Fig. 3. High resolution image of a stack layers and zoom on a $\mathrm{TCO} / \mathrm{TiO}_{2}$ interface region after reducing the size of $\mathrm{TiO}_{2}$ particles.

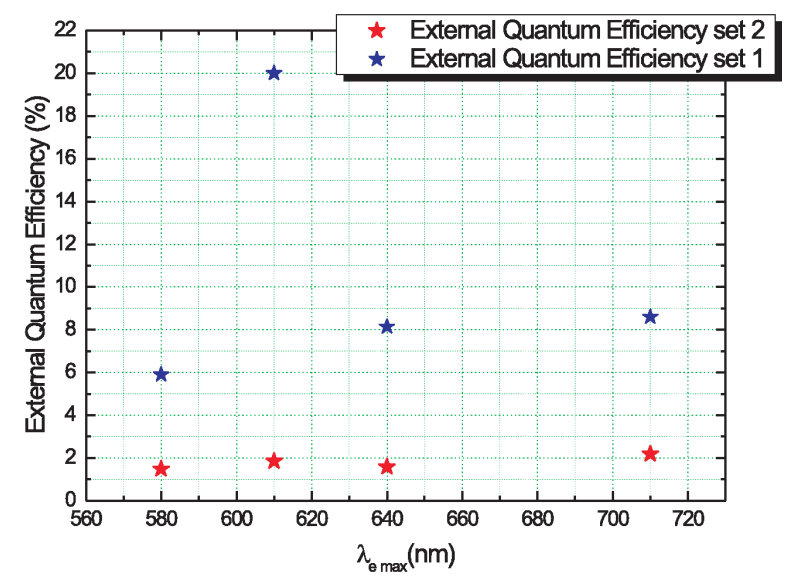

Fig. 4. External quantum efficiency vs. wavelength for four different samples made using reduced $\mathrm{TiO}_{2}$ particles.

notice improvement of EQE by at least 300\%. Since, no other parameters were changed when fabricated the samples beside the size of the $\mathrm{TiO}_{2}$ particles size, this improvement is directly related to the improvement of the interface between $\mathrm{TCO} / \mathrm{TiO}_{2}$.

\section{Conclusions}

Chemical strengthening process during that deeper penetration of the glass surface by ions contained in the brine bath takes place, contributes to the hardness of the glass sheets, which reduces the occurrence of surface defects that may cause reflections in ultrathin glass. This type of glass is perfect substrate for deposition of nanomaterials, i.e. conductive films. What is more, ultrathin glass significantly reduce the weight of the whole photovoltaic panel structure relative to the solutions currently common in photovoltaic. General conclusion is that thanks to good flexibility, smooth surface, good transmittance, excellent gas and water barrier, much higher toughened in relations to thermally tempered glass, higher impact resistance, increased corrosion resistance and much higher abrasion rate, ultrathin glass is ideal material in photovoltaic applications.

\section{Summary}

In this work we characterized the interface of heterostrucure $\mathrm{TCO} / \mathrm{TiO}_{2}$. We showed that by reducing the size of $\mathrm{TiO}_{2}$ particles it is possible to improve the interface between transparent conductive oxide and titanium dioxide layer. Better charge transfer will eventually give higher solar efficiency. By this easy and cheap way we could successfully achieve the improvement of the external efficiency by about $300 \%$.

\section{Acknowledgments}

This work is supported by The National Centre for Research and Development (Grant No. POIR.01.01.0100-0598/15).

\section{References}

[1] Climate Change Secretariat (UNFCCC), Uniting on climate, A guide to the Climate Change Convention and the Kyoto Protocol, UNEPGRID-Arendal 9, (2012).

[2] B. O'Regan, M. Grätze, Nature 252, 737 (1991).

[3] A.J. Nozik, Physica E 14, 115 (2002).

[4] D. Chen, F. Huang, Y.-B. Cheng, R.A. Caruso, Adv. Mater. 21, 2206 (2009).

[5] S. Nakade, Y. Saito, W. Kubo, T. Kitamura, Y. Wada, S. Yanagida, J. Phys. Chem. B 107, 8607 (2003).

[6] A. Hakki, R. Dillert, D. Bahnemann, Catal. Today 144, 154 (2009).

[7] J. Zhang, K. Xu, Appl. Surf. Sci. 221, 1 (2001). 